\title{
Formación de la Aleación Zn-21.6\%Al-2.0\%Cu por Pulvimetalurgia: Efecto del Lubricante en las Briquetas
}

\author{
José A. Aragón \\ Univ. Autónoma Metropolitana, Unidad Azcapotzalco, Departamento de Materiales, \\ Avda. San Pablo № 180, Col. Reynosa Tamaulipas, 02200 México, D.F.-México \\ (e-mail: alja@correo.azc.uam.mx)
}

\section{Resumen}

En este trabajo se presenta la elaboración de la aleación $\mathrm{Zn}-21.6 \% \mathrm{Al}-2.0 \% \mathrm{Cu}$ (porcentajes en masa), por un método de metalurgia de polvos y se analiza el efecto de distintos lubricantes, usados en las briquetas. Se elaboraron mezclas de polvos de $\mathrm{Zn}$, Al y $\mathrm{Cu}$ con acetona, cera y ácido esteárico como lubricantes, y se compactaron uniaxialmente a una presión de $77.4 \mathrm{MPa}$. Las briquetas se sinterizaron bajo un flujo de argón y en intervalos de 15 minutos a $357^{\circ} \mathrm{C}$. Los materiales fueron caracterizados estructuralmente con microscopio óptico, ensayos de microdureza Vickers, dureza Rockwell $\mathrm{F}$ y difracción de rayos $\mathrm{X}$. El ácido esteárico y la cera funcionan similarmente como lubricantes y la acetona retarda la formación de la aleación, pero causa mayores durezas, luego del sinterizado. La aleación se forma totalmente cuando el lubricante es cera y se sinteriza más de 45 minutos. La rapidez de formación de la aleación depende del tipo de lubricante en las briquetas.

Palabras clave: metalurgia de polvos, aleación Zn-21.6\%Al-2.0\%Cu, caracterización estructural, sinterizado

\section{Formation of Zn-21.6\%Al-2.0\%Cu Alloy by Powder Metal- lurgy: Effect of the Lubricant in Briquettes}

\begin{abstract}
In this work the elaboration of $\mathrm{Zn}-21.6 \% \mathrm{Al}-2.0 \% \mathrm{Cu}$ alloy (mass percentages), prepared by a method of powder metallurgy and the effect of different lubricants used in the briquettes were studied. Mixtures of powders of $\mathrm{Zn}, \mathrm{Al}$ and $\mathrm{Cu}$ with acetone, wax and stearic acid as lubricants were prepared and compacted up to a pressure of $77.4 \mathrm{Mpa}$. The briquettes were sintered under argon flux and at intervals of 15 and 45 minutes at $357^{\circ} \mathrm{C}$. The materials were structurally characterized by optical microscopy, micro-hardness Vickers, hardness Rockwell $F$ tests and X-rays diffraction. The stearic acid and wax work similar to lubricants and acetone slows down the formation of the alloy, but it causes greater hardnesses after sintering. The alloy is completely formed when the lubricant is wax and the sintering time is more than 45 minutes. The velocity of formation of the alloy depends on the type of lubricant in the briquettes.
\end{abstract}

Keywords: powder metallurgy, Zn-21.6\%Al-2.0\%Cu alloy, structural characterization, sintering 


\section{INTRODUCCIÓN}

Las aleaciones ricas en $\mathrm{Zn}$, con alto contenido de $\mathrm{Al}$ y modificadas o no con elementos de aleación, han sido estudiadas con mucho interés en los últimos años (Chen et al., 2004; Hirata et al., 2007). Entre estas aleaciones destacan las elaboradas a base de la liga eutectoide $\mathrm{Zn}-22 \% \mathrm{p} \mathrm{Al}$, que es interesante porque presenta super-plasticidad (Backofen et al., 1964). Algunas de estas aleaciones se denominan ZA y tienen características similares a las de algunos bronces, por lo que son útiles en aplicaciones que requieren resistencia al desgaste bajo carga (kubel, 1987; Purcek et al., 2007).

La producción masiva de piezas con formas y dimensiones cercanas a las de su uso en tiempos relativamente cortos, para esas aplicaciones, se logra con el proceso pulvimetalúrgico $(\mathrm{P} / \mathrm{M})$. Es importante entonces el estudio del comportamiento y de las propiedades resultantes de la aleaciones elaboradas en base a la aleación $\mathrm{Zn}-22 \%$ p Al. El proceso P/M fue aplicado a esta aleación eutectoide binaria por Aragón et al. (2001), y Xun y Mohamed (2004); y a la misma modificada con 2\% (en peso) de Cu - usando polvos de esta aleación - por Martínez y Torres (1997), y Aragón y Villegas (2006).

En este trabajo se elaboraron mezclas de $\mathrm{Zn}-21.6 \% \mathrm{p} \mathrm{Al}-2.0 \% \mathrm{p}$ Cu con distintos lubricantes, para establecer el efecto que tienen en la formación y las propiedades mecánicas básicas de la aleación con la misma composición y elaborada por la técnica P/M. Se realizó el seguimiento de los cambios estructurales y de las propiedades mecánicas básicas y análisis de rayos $\mathrm{X}$ en las mezclas en función del tiempo de su sinterizado. Los resultados servirán para optimizar la elaboración por P/M y las propiedades de materiales compuestos con esta aleación como matriz y reforzada con cerámicos.

\section{PROCEDIMIENTO EXPERIMENTAL}

Se usaron polvos de $\mathrm{Zn}, \mathrm{Al}$ y $\mathrm{Cu}$ de pureza grado reactivo y con tamaños de partículas menores a 45 $\mu \mathrm{m}$. Acetona, ácido esteárico y cera se emplearon como lubricantes, pues se queman un porcentaje grande en un intervalo estrecho de temperatura, menor a la temperatura de fusión del Zn (Klar, 1984). Se pesaron muestras de polvos de $20 \mathrm{~g}$, con fracciones en peso de $76.44 \%$ de $\mathrm{Zn}, 21.6 \% \mathrm{Al}$ y $2.0 \% \mathrm{Cu}$, y $0.78 \%$ (de $20 \mathrm{~g}$ ) de cada lubricante: el porcentaje de lubricante se eligió dentro del intervalo de cantidades - $0.5 \%$ a $1.5 \%$ - usualmente empleadas que reporta Klar (1984). Se añadieron por separado los lubricantes sólidos a las muestras de polvos y cada una se mezcló: vibrando y girando, de arriba hacia abajo y viceversa, la bolsa contenedora; este procedimiento ya ha sido usado (Aragón et al. 2001; Aragón y Villegas 2006) y produce mezclas homogéneas. La acetona se vació sobre las muestras con una jeringa poco antes de compactarlas. Las mezclas se compactaron uniaxialmente con carga creciente hasta una presión de $74 \mathrm{MPa}$, manteniéndola durante 2 minutos y extrayendo después las briquetas con la misma prensa: la acetona escurría de las briquetas compactadas con este lubricante; los compactos en verde tenían un diámetro de $25.4 \mathrm{~mm}$ y altura de $7 \mathrm{~mm}$.

El sinterizado de las briquetas se realizó en etapas de 15 minutos de duración, en un horno programado en segmentos, bajo un flujo de argón de $0.28 \mathrm{~m}^{3} / \mathrm{h}$ y a la temperatura de $(357 \pm 1)^{\circ} \mathrm{C}$ : el equilibrio térmico en la cámara del horno se garantizó realizando su calibración previa y automática, empleando una carga de peso similar al de las muestras y con el argón fluyendo; los intervalos de 15 minutos de sinterizado se eligieron considerando que la mayoría de los metales no ferrosos se sinterizan adecuadamente entre los 45 y 90 minutos. El enfriamiento de las probetas se hizo en el horno.

Una cara de cada probeta fue desbastada con papeles abrasivos de distintas granulometrías y pulida con una suspensión de alúmina de 0.05 micrones y alcohol etílico, se sumergió después en una solución de $99.5 \mathrm{ml}$ de alcohol y $0.5 \mathrm{ml}$ de ácido nítrico para revelar su estructura

Las estructuras fueron analizadas y fotografiadas en un microscopio metalográfico PMG3 de Olympus. Se midieron las durezas Rockwell F (DRF) de las muestras y las microdurezas Vickers (DV) de sus componentes (carga de prueba de $10 \mathrm{~g}$ ), siguiendo para esto las normas de la ASTM E 18 (2007) y E 384 (2008), respectivamente, con los equipos Micromet 2003 de Buehler y Missawa Seiki. Finalmente, se realizaron análisis de rayos $X$ en las probetas y para cada tiempo de sinterizado por medio de un difractómetro $D-500$ de Siemens, empleando la radiación de $\mathrm{Cu}(\lambda \mathrm{K} \alpha=15.4 \mathrm{~nm})$. 
Tabla 1: Componentes en las Estructuras según el Lubricante y el Tiempo de Sinterizado.

\begin{tabular}{|c|l|l|l|}
\hline $\begin{array}{c}\text { Tiempo de sinterizado } \\
\text { (minutos) }\end{array}$ & \multicolumn{3}{|c|}{ Lubricante } \\
\cline { 2 - 4 } & \multicolumn{1}{|c|}{ Acetona } & \multicolumn{1}{|c|}{ Acrawax } & \multicolumn{1}{|c|}{ Ácido esteárico } \\
\hline Briqueta & Zn, Al, Cu y Po & Zn, Al, Cu y Po & Zn, Al, Cu y Po \\
\hline 15 & ZC, ZG, Cu y Po & ZC, ZG Cu y Po & ZC, ZG Cu y Po \\
\hline 30 & ZC, ZG, Po, Pl y (Cu en Zn) & ZC, ZG, Po, PF & ZC, ZG, Po y PF \\
\hline 45 & ZC, ZG, Po, Pl, PF y (Cu en Zn) & ZC, ZG, Po y PF & ZC, ZG, Po y PF \\
\hline 60 & ZC, ZG, Pl, PF y PG & PF, PG y Cl & ZC, ZG, PF y Cl \\
\hline 75 & ZC, ZG, Pl, PF y PG & PF, PG y Cl & ZC, ZG, PF, PG y Cl \\
\hline
\end{tabular}

\section{RESULTADOS Y DISCUSIÓN}

El reactivo empleado para revelar las estructuras imparte tonalidad clara a los componentes ricos en Zn; gris, a los abundantes de Al, y los de cobre conservan su color original. Los componentes estructurales, según el tipo de lubricante y el tiempo de sinterizado, se presentan en la tabla 1. El significado de las siglas en la tabla es: ZC, zonas claras lisas y ricas en Zn; ZG, zonas grises y ricas en Al; PI, regiones con subestructura tipo perlita inicial, caracterizada por glóbulos sobre un constituyente liso; $\mathrm{PF}$, perlita fina; $\mathrm{PG}$, perlita gruesa; $\mathrm{Cl}$ : islas claras, lisas y brillantes de un compuesto intermetálico; (Cu en $\mathrm{Zn}$ ), islas de $\mathrm{Zn}$ con centro de $\mathrm{Cu}$, y Po, poros.

Las estructuras de las briquetas fueron similares en general, sin importar el tipo de lubricante (Fig. 1). Su diferencia fundamental radicó en el grado de su porosidad, Po, que fue de mayor a menor proporción en los compactos formados con acetona, cera y ácido esteárico; este atributo decreció continuamente con el incremento del tiempo de sinterizado en la muestra compactada con acetona. En cambio, en las probetas compactadas con los lubricantes sólidos, la porosidad incrementó luego de sinterizarlas 15 minutos, observándose una mayor porosidad en la muestra elaborada con cera, y disminuyó posteriormente al incrementar el tiempo de su sinterizado.

El incremento de la porosidad en las muestras formadas con los lubricantes sólidos, se debió a la combustión completa o evaporación de éstos durante la primera etapa del sinterizado, causando la disminución de la dureza Rockwell $F$ (DRF). En cambio, la muestra elaborada con acetona y sinterizada 15 minutos registró un incremento de la DRF, que fue la mayor obtenida (Fig. 2).

La máxima dureza que presentó la probeta formada con acetona y sinterizada durante 15 minutos, y las durezas Vickers (DV) obtenidas similares en los componentes de ésta y su briqueta (Fig. 3), infor man que ocurrió la consolidación temprana de la mezcla de polvos por la unión de sus componentes por difusión, lo cual impidió que los componentes se deformaran plásticamente en forma conjunta.

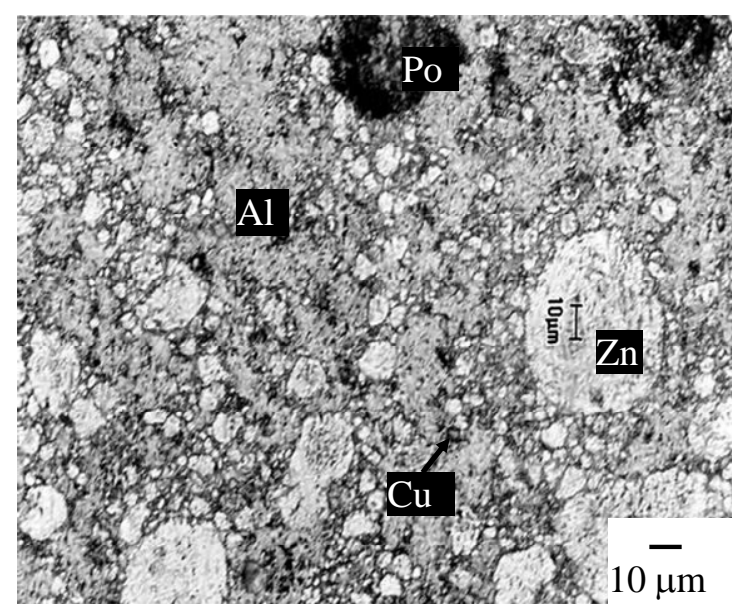

Fig. 1: Micrografía de la briqueta compactada con ácido esteárico.

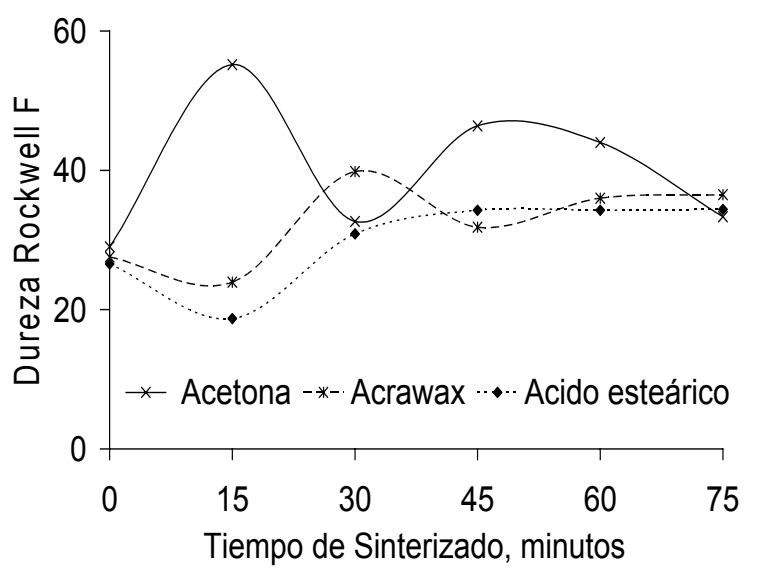

Fig. 2: Dureza Rockwell F de las muestras en función del tiempo de su sinterizado y del tipo de lubricante usado en la compactación. 


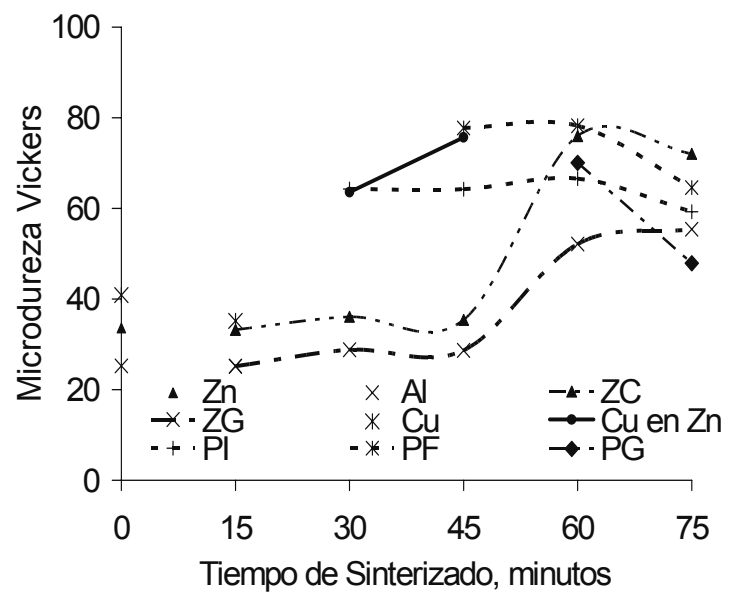

Fig. 3: Microdureza Vickers de los componentes en función del tiempo de sinterizado en la muestra formada con acetona (carga $10 \mathrm{~g}$ ).

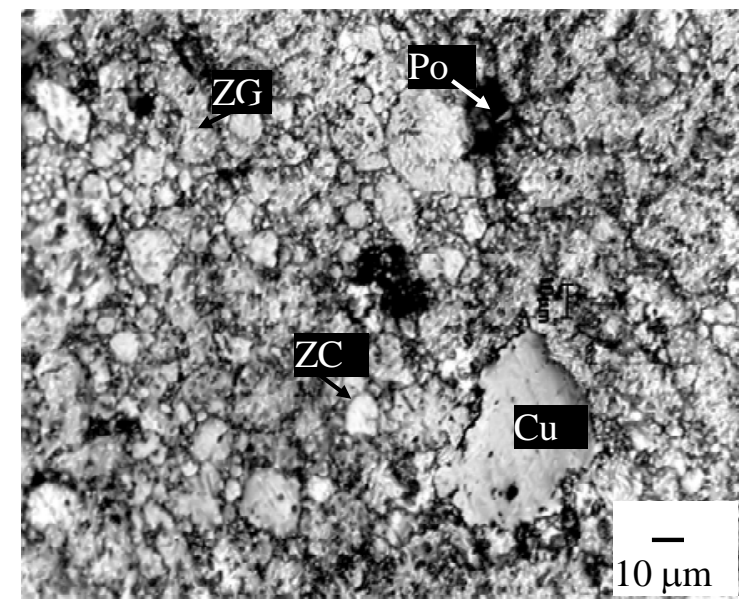

Fig.4: Región representativa de las estructuras de las muestras sinterizadas durante 15 minutos.

Esta consolidación no estuvo asociada con la presencia de componentes estructurales nuevos en esta probeta: su estructura fue parecida a las de muestras formadas con los lubricantes sólidos (Fig. 4). Las estructuras de las muestras formadas con cera y esteárico, y sinterizadas 30 y 45 minutos fueron similares, caracterizadas por la presencia de ZC, ZG y/o PF (Fig. 5). En cambio, las estructuras de las muestras elaboradas con acetona y sinterizadas los mismos intervalos de tiempo, exhibieron además regiones de $\mathrm{PI}$ e islas (Cu en $\mathrm{Zn}$ ).

La presencia de zonas con la subestructura del tipo perlita fina, confirma que la aleación se formó en ciertas regiones de las probetas formadas con los lubricantes sólidos y sinterizadas entre los 15 y 30 minutos. La fracción de la aleación se incrementó con el tiempo de sinterizado - 30 a 45 minutos -. En cambio, la aleación se produjo a tiempos posteriores, entre los 30 y 45 minutos, en algunas regiones de la muestra conformada con acetona: en esta probeta fue más lenta la difusión de las especies atómica, y tenía regiones de componente tipo perlita mal formada, $\mathrm{PI}$, e islas (Cu en Zn) (Fig. 6).

Por otro lado, la formación de la aleación o de regiones con la subestructura PF, estuvo relacionada con un incremento de las DRF en las probetas, no importando la clase de lubricante usado en la com pactación. Pero, el aumento del grado de formación de la aleación estuvo asociado con la reducción de la DRF de la muestra conformada con cera, y con un incremento adicional y ligero de la dureza en la probeta formada con esteárico, entre los 30 y 45 minutos de proceso: de manera que las durezas de ambas muestras fueron casi las mismas, y de magnitudes mucho menores que la dureza de la probeta formada con acetona, a los 45 minutos de proceso (Fig. 2).

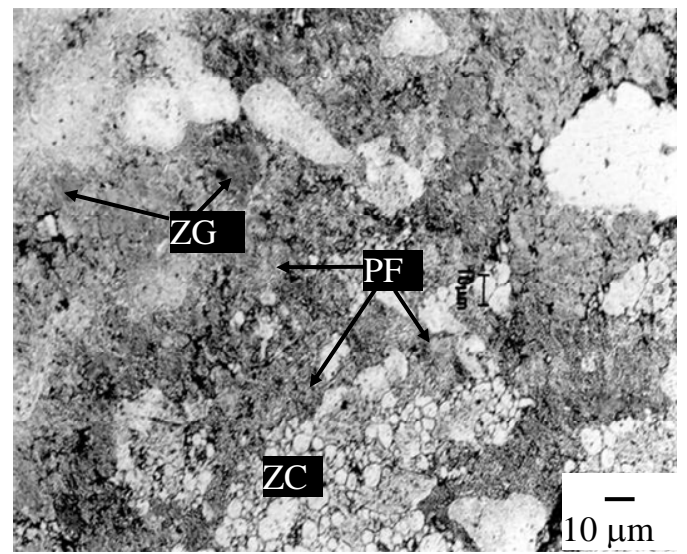

Fig. 5: Región representativa de las estructuras de las muestras formadas con cera y ácido esteárico, sinterizadas durante 30 minutos.

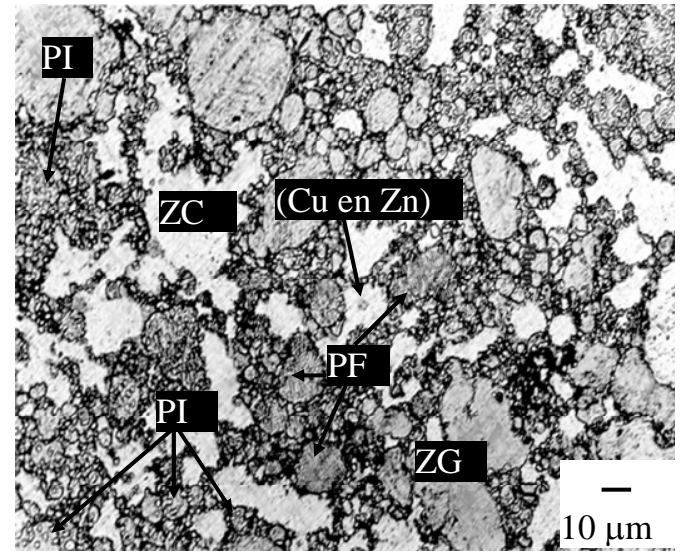

Fig. 6: Zona representativa de la estructura de la probeta compactada con acetona y sinterizada durante 45 minutos. 


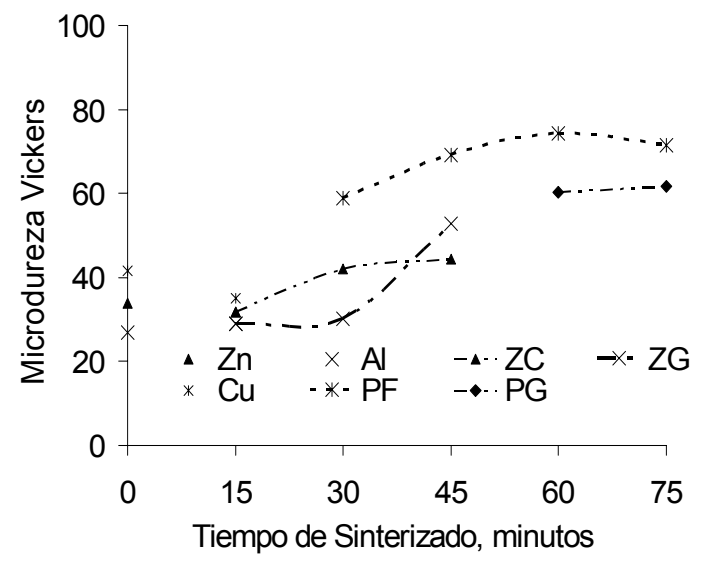

Fig. 7: Microdureza Vickers de los componentes de la muestra compactada con cera, en función del tiempo de sinterizado (carga $10 \mathrm{~g}$ ).

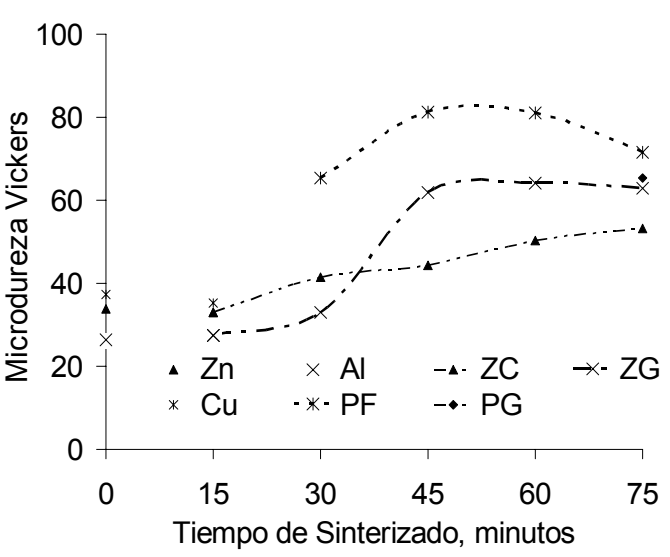

Fig. 8: Microdureza Vickers de los componentes de la probeta formada con esteárico, en función del tiempo de sinterizado (carga $10 \mathrm{~g}$ ).

Las variaciones de DRF en las probetas conformadas con acetona o cera y procesadas entre los 15 y 45 minutos, no concordaron con los cambios de la dureza de sus componentes estructurales, porque la mayoría de ellos se endureció progresivamente en este intervalo de tiempo (Figs. 3 y 7). Mientras que el incremento progresivo de la DRF en la probeta formada con ácido y sinterizada entre los 15 y 45 minutos, si estuvo relacionado con el de la dureza de sus constituyentes (Fig. 8).

Las estructuras de la probeta compactada con cera y procesada 60 y 75 minutos, eran casi totalmente un conjunto de colonias del componente tipo perlita bien definida (Fig. 9), que embebían algunas islas que, por su alta dureza - entre 180 y 240 unidades Vickers -, eran de un compuesto intermetáli$\mathrm{co}, \mathrm{Cl}$ : los diagramas de rayos $\mathrm{X}$ obtenidos para estas estructuras, no presentados aquí, informaron que los $\mathrm{Cl}$ eran de fase $\varepsilon, \mathrm{CuZn}$, tal y como se informa en el corte isotérmico en $20^{\circ} \mathrm{C}$ del sistema ternario $\mathrm{Zn}-\mathrm{Al}-\mathrm{Cu}$ (Villars et al., 1995). Entonces, la aleación $\mathrm{Zn}-21.6 \% \mathrm{p} \mathrm{Al}-2.0 \% \mathrm{p}$ Cu se forma totalmente al emplear la cera en la elaboración de las briquetas y a tiempos de sinterizado entre los 45 y 60 minutos. En cambio, las estructuras de la probeta formada con ácido esteárico o acetona y sinterizada 60 y 75 minutos, estuvieron integradas, entre otros constituyentes, por ZC y ZG (Fig. 10). Luego, estos lubricantes retardan la formación de la aleación, mucho más la acetona: porque las PI estuvieron sólo en la muestra formada con este lubricante, y las cantidades de sus PF y PG fueron menores que las de componentes similares en las muestra compactada con ácido esteárico.

La DRF incremento continua y ligeramente en la muestra formada con cera, no varió en la conformada con ácido esteárico, y disminuyó progresiva y considerablemente en la compactada con acetona, luego de sinterizarlas por arriba de los 45 y hasta los 75 minutos. Estas variaciones podrían deberse a los cambios en la intensidad de unión por difusión entre los componentes estructurales.

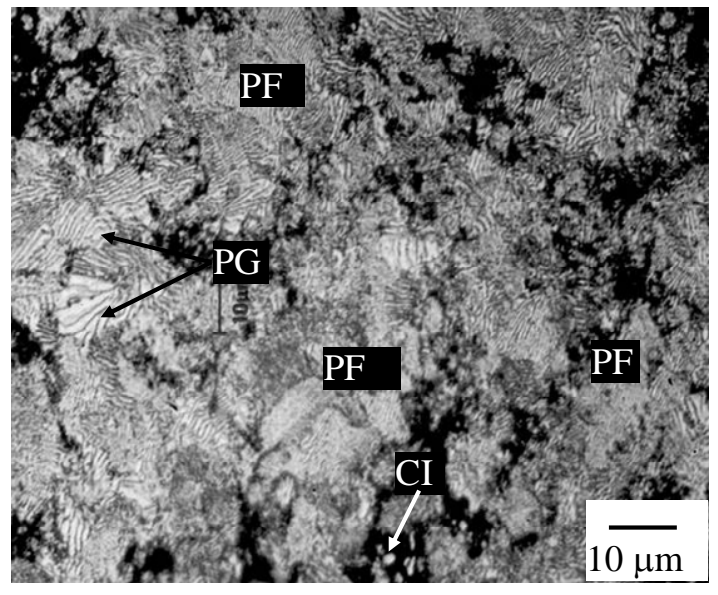

Fig. 9: Región de la muestra formada con cera y sinterizada 75 minutos.

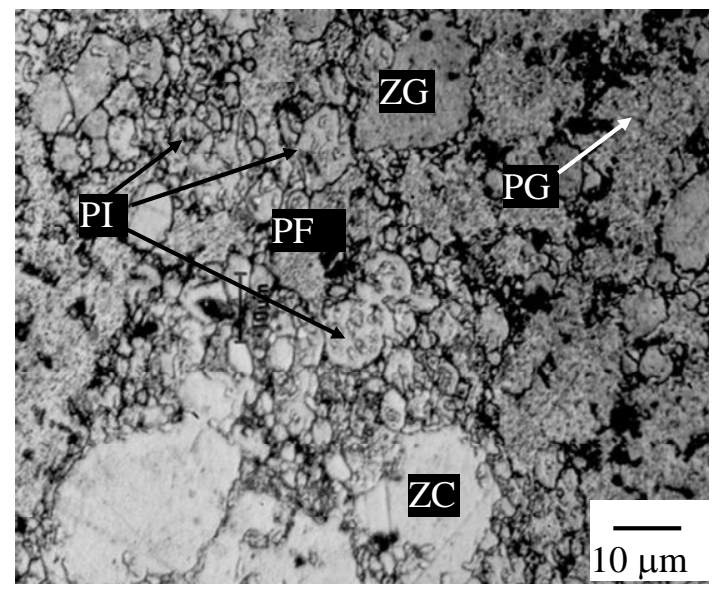

Fig. 10: Región de la probeta compactada con acetona y sinterizada 75 minutos. 
Los resultados de este trabajo son en general diferentes a los reportados por Aragón y Villegas (2001) para la aleación $\mathrm{Zn}-22 \%$ p Al sin Cu, elaborada por P/M: donde esta aleación se forma totalmente a los 60 minutos de sinterizado en la muestra conformada con ácido esteárico.

\section{CONCLUSIONES}

De acuerdo a los resultados se concluye que el ácido esteárico y la cera funcionan similarmente como lubricantes; las durezas de las probetas formadas con éstos lubricantes son similares durante casi todo el proceso de sinterizado; la acetona retarda la formación de la aleación, pero causa mayores durezas después del sinterizado durante 15 y 45 minutos; la cera causa que se forme la aleación en baja proporción en tiempos breves, y totalmente entre los 45 y 60 minutos. El tipo de lubricante en la compactación influye en la rapidez de formación de la aleación por P/M.

\section{AGRADECIMIENTOS}

Se agradece al jefe y personal del área de Construcción de la UAM - Azcapotzalco, el préstamo de la prensa Forney y la asesoría recibida.

\section{REFERENCIAS}

Aragón, J.A., J.C. Villegas y A.S. De Ita; Elaboración de la Aleación Zn - 22\%p Al a partir de Polvos Metálicos. Información Tecnológica: 12(3), 113-120 (2001).

Aragón, J.A. y J.C. Villegas; Efectos del 2\%p Cu en la Respuesta ante el Sinterizado de la Mezcla de Polvos Zn - 22\%p Al, Información Tecnológica: 17(6), 51-56 (2006).

Backofen, W.A., I.R. Turner y D.H. Avery; Superplasticity in an Al Zn Alloy, Transactions of the ASM: 57, 980-990 (1964).

Chen, H., X. Xin, Y.P. Ren y S.M. Hao; Phase Diagram of Al-Zn-Cu System in low Cooper Side at Room Temperature, Acta Metallurgica Sinica: 40(8), 841-845 (2004).

ASTM E 92; Test Methods for Rockwell Hardness of Metallic Materials, ASTM International, West Conshohocken, PA, http:///www.astm.org. (2007)

ASTM E 384; Test Method for Microindentation Hardness of Materials, ASTM International, West Conshohocken, PA, http:///www.astm.org. (2008)

Hirata, T. y otros cuatro autores; Relationship between Deformation Behaviour and Microstructural Evolution of Friction Stir Processed Zn-22wt\%Al Alloy, Scripta Materialia: 56(6), 477-480 (2007).

Klar, E.; Powder Metallurgy, Metals Handbook, 9ª edición, 7, 190-193, ASM Inter, Ohio-EUA (1984).

Kubel, E.J.; Expanding Horizons for ZA Alloys. Advanced Materials \& Process Inc. Metal Progress: 132(1), 51-57 (1987).

Martínez, E. y G. Torres; Compresión Ductility of Zn-21Al-2Cu Prepared by Powder Metallurgy Techniques, Materials \& Design: 18(3), 127-130 (1997).

Purcek, G. y otros 5 autores; Enhancement in Mechanical Behavior and Wear Resistance of Severe Plastically Deformed Two-Phase Zn-Al Alloys, Inter. J. of Materials Research: 98(4), 332-338 (2007).

Villars P., A. Price y H. Okamoto; Ternary Alloy Phase Diagrams, 3, 3416, ASM International, OhioUSA (1995).

Xun, Y. y F.A. Mohamed; Superplastic Behavior of Zn-22\%Al Containing a Nano-Scale Dispersion Particles, Acta Materialia: 52(15), 4401-4412 (2004). 